\title{
DIFERENÇA ENTRE MACHOS SUÍNOS NA MANUTENÇÃO DA VIABILIDADE ESPERMÁTICA A $17^{\circ} \mathrm{C}$
}

\author{
DIFFERENCE AMONG BOARS IN THE MAINTENANCE OF SPERM VIABILITY AT $17^{\circ} \mathrm{C}$
}

\author{
Goreti Ranincheski dos Reis ${ }^{1}$, Mari Lourdes Bernardi ${ }^{2}$, Patrícia Schwarz ${ }^{3}$, \\ Fernando Pandolfo Bortolozzo ${ }^{4}$ Ivo Wentz ${ }^{4}$
}

\begin{abstract}
RESUMO
Machos suínos apresentam variação no tempo de manutenção da motilidade (MOT), durante o resfriamento do sêmen. A identificação de doadores que possuam sêmen viável por período mais longo durante o armazenamento a $17^{\circ} \mathrm{C}$, permitiria seu uso estratégico em programas de inseminação. O objetivo deste estudo foi verificar se cachaços selecionados conforme o tempo de manutenção da motilidade espermática a $17^{\circ} \mathrm{C}$, apresentavam o mesmo perfil em coletas subseqüentes, além de avaliar o comportamento das variáveis acrossomas normais (NAR) e membranas íntegras (MI) durante o armazenamento. Cinco ejaculados de 30 machos foram analisados conforme a manutenção da MOT a $17^{\circ} \mathrm{C}$, sendo classificados em três tipos: MOT $<60 \%$ nas $72 \mathrm{~h}(\mathrm{EI})$; MOT $\geq 60 \%$ nas $72 \mathrm{~h}$ e $<60 \%$ nas $144 \mathrm{~h}$ (EII) e MOT $\geq 60 \%$ nas 144h (EIII). Doze machos foram selecionados e distribuídos em três grupos: MAIOR, MÉDIA e MENOR sensibilidade espermática ao resfriamento. O critério utilizado foi que no mínimo 4/5 dos ejaculados fossem classificados como EI para MAIOR, EII para MÉDIA e EIII para MENOR. Após a seleção, foram coletados cinco ejaculados de cada macho, sendo a MOT avaliada a cada 24h, e NAR e MI nas 24, 72, 120 e $168 \mathrm{~h}$. O coeficiente de variação foi menor para MI e NAR do que para MOT. Diferenças entre machos foram observadas das 24 até $168 \mathrm{~h}$ para MOT, nas 120 e $168 \mathrm{~h}$ para MI e nas $72 \mathrm{e}$ $168 \mathrm{~h}$ para NAR. Machos menos sensíveis ao resfriamento apresentaram menor variação nas características dos ejaculados do período pré- para o pós-seleção. Diferenças observadas entre cachaços na MOT nem sempre são acompanhadas por diferenças na integridade da membrana espermática e do acrossoma.
\end{abstract}

Descritores: motilidade, resfriamento, sêmen, suíno.

\begin{abstract}
Boars show a variability in the time of motility (MOT) maintenance during sperm cooling. Identification of boars that retain the viability of semen for longer periods during storage at $17^{\circ} \mathrm{C}$, would allow their strategic use in programs of insemination. The objective of this study was to verify if boars selected according to the maintenance of sperm motility (MOT), at $17^{\circ} \mathrm{C}$, showed the same pattern on subsequent collections and to evaluate the behaviour of normal acrosomes (NAR) and plasma membrane integrity (MI) during storage. Five ejaculates from 30 boars were evaluated according to the time of maintenance of MOT at $17^{\circ} \mathrm{C}$, and they were classified in three types: MOT $<60 \%$ at $72 \mathrm{~h}$ (EI); MOT $\geq 60 \%$ at $72 \mathrm{~h}$ and $<60 \%$ at $144 \mathrm{~h}$ (EII) and MOT $\geq$ $60 \%$ at $144 \mathrm{~h}$ (EIII). Twelve boars were selected and classified, in three groups: HIGH, INTERMEDIATE and LOW chilling sensitivity. The criterion used was that a minimum of 4 from 5 ejaculates was classified as EI for HIGH, EII for INTERMEDIATE and EIII for LOW groups. After selection, five ejaculates from each boar were collected and MOT was evaluated each 24h, MI and NAR at 24, 72, 120 and 168h of storage. The coefficient of variation of MI and NAR was lower than that of MOT. Differences among males for MOT were observed from 24 up to $168 \mathrm{~h}$, at 120 and $168 \mathrm{~h}$ for MI and at 72 and $168 \mathrm{~h}$ for NAR. Males selected as being less sensitive to cooling showed a lower variability in the characteristics of their ejaculates, between pre- and post-selection periods. Differences among males for motility were not always followed by differences in the plasmatic membrane and acrosomal integrity.
\end{abstract}

Key words: chilling, motility, semen, swine.

${ }^{1}$ Programa de Pós-Graduação em Ciências Veterinárias (PPGCV), Faculdade de Veterinária (FAVET) de Porto Alegre, Universidade Federal do Rio Grande do Sul (UFRGS). ${ }^{2}$ Departamento de Zootecnia, Faculdade de Agronomia (UFRGS). ${ }^{3}$ Acadêmica da FAVET - UFRGS. ${ }^{4}$ Setor de Suínos, FAVET - UFRGS. CORRESPONDÊNCIA: G. R. Reis [e-mail: grreis@ hotmail.com]. 


\section{INTRODUÇÃO}

Durante o armazenamento in vitro do sêmen suíno é observada uma queda gradativa na motilidade espermática. A diferença entre machos quanto à sensibilidade ao processo de resfriamento já foi reportada em suínos [15, 17], existindo machos cuja motilidade espermática é mantida por um período maior de tempo, enquanto outros apresentam uma queda mais precoce [22]. O sêmen de doadores que mantêm a viabilidade espermática in vitro por um maior período de tempo, poderia ser utilizado em situações estratégicas de inseminação [3].

A fertilidade do machoé de difícil predição, sendo sugerida [19] a análise combinada de diversos parâmetros seminais para possibilitar uma estimativa mais exata do potencial fecundante do sêmen. No entanto, por ser a fertilidade comprometida com valores de motilidade abaixo de 60\% [7], este valor é usualmente utilizado como referência para a utilização das doses de sêmen na inseminação artificial (IA) de suínos.

A estimativa visual da motilidade [5] e das alterações morfológicas $[18,25]$ têm sido comumente empregadas para avaliar a qualidade seminal. Também têm sido empregadas colorações fluorescentes para avaliar a integridade das membranas [12, 25]. Essas colorações, apesar de não serem de execução difícil, necessitam de equipamentos onerosos e de técnicos treinados, o que ainda restringe sua utilização ao campo experimental.

Este estudo teve como objetivo verificar se cachaços selecionados conforme o tempo de manutenção da viabilidade espermática durante o armazenamento a $17^{\circ} \mathrm{C}$, através da avaliação da motilidade, mantinham este padrão ao longo das coletas, e para avaliar o comportamento dos parâmetros acrossomas normais e membranas íntegras, ao longo do armazenamento e das coletas.

\section{MATERIAIS E MÉTODOS}

Foram utilizados, como doadores de sêmen, machos suínos selecionados do plantel de 170 animais de uma central de IA (CIA). A genética utilizada foi de uma linhagem comercial constituída pelas raças Large White, Landrace e Pietrain. A frequiência de coletas dos machos, praticada como rotina na CIA, era de uma coleta a cada sete dias, entre sete e dez meses de idade, três coletas a cada 14 dias dos 11 aos 12 meses e duas coletas a cada sete dias a partir dos 13 meses.

Inicialmente, foi realizada a avaliação de 30 machos suínos, durante os meses de janeiro a maio, com um intervalo médio de duas semanas entre as análises. Foi avaliada a motilidade (MOT) de cinco ejaculados destes 30 machos, por um período de sete dias, a $17^{\circ} \mathrm{C}$. Os ejaculados foram classificados em três grupos, de acordo com o período de manutenção da MOT, sendo: MOT $<60 \%$ nas $72 \mathrm{~h}$ (EI); MOT $\geq 60 \%$ nas $72 \mathrm{~h}$ e $<60 \%$ nas $144 \mathrm{~h}$ (EII) e MOT $\geq 60 \%$ nas 144h (EIII). Dos 30 machos, foram selecionados 12, os quais tinham entre 19 e 22 meses de idade. Os machos foram considerados como de maior (MAIOR), média (MEDIA) e menor (MENOR) sensibilidade espermática ao resfriamento. O critério utilizado foi que dos 5 ejaculados no mínimo 4 fossem classificados como EI para MAIOR, EII para MEDIA e EIII para MENOR, respectivamente. Todos os ejaculados dos machos selecionados apresentaram valores de MOT acima de $80 \%$, na avaliação pós-diluição do sêmen.

Foram avaliados cinco ejaculados de cada um dos machos selecionados (3, 4 e 5 machos no grupo MAIOR, MEDIA e MENOR, respectivamente), durante o período de junho a setembro, com um intervalo médio de três semanas entre as análises. As amostras de sêmen, contendo três bilhões de espermatozóides em diluente BTS (Beltsville Thawing Solution), foram armazenadas a $17^{\circ} \mathrm{C}$. A avaliação da MOT foi efetuada a cada $24 \mathrm{~h}$ durante sete dias de armazenamento. Os percentuais de acrossomas normais (NAR) [18] e de membranas íntegras foram avaliados nas 24, 72, $120 \mathrm{e} 168 \mathrm{~h}$ de armazenamento. A integridade das membranas (MI) foi avaliada por coloração com diacetato de carboxifluoresceína e iodeto de propídio $[9,16]$.

Para a comparação entre os machos, os dados referentes à MOT, NAR e MI foram submetidos à análise de variância pelo procedimento GLM [20]. Quando o efeito da semana de coleta foi significativo esta permaneceu no modelo. Os dados foram submetidos à transformação arco seno raiz quadrada, antes de serem submetidos à análise, mas as médias não transformadas são apresentadas nos resultados. Foram avaliadas as relações entre os parâmetros estudados pela correlação de Spearman [20].

\section{RESULTADOS}

Na Tabela 1 está a distribuição do número de machos conforme o número de ejaculados de cada tipo. O número de machos com 4 ou mais ejaculados de cada tipo foi menor para os dois extremos de sensibilidade (EI e EIII), havendo uma maior concentração de machos no grupo de sensibilidade média (EII).

Na Tabela 2 está a distribuição do número de ejaculados de cada tipo verificados em cada macho selecionado, antes e após a seleção. Em 3 machos, foram coletados 4 ejaculados ao invés de 5 , no perío- 
do pós-seleção. Os machos selecionados como sendo mais sensíveis ao resfriamento (Grupo MAIOR) apresentaram alguns ejaculados de sensibilidade média (tipo EII), com apenas 1 dos 15 ejaculados coletados sendo de menor sensibilidade (tipo EIII). Machos considerados menos sensíveis ao resfriamento (Grupo MENOR) não apresentaram nenhum ejaculado de maior sensibilidade (tipo EI), sendo os que apresentaram menor mudança de perfil no período pós-seleção em relação ao pré-seleção. A maior variação foi observada nos machos de sensibilidade média (Grupo MÉDIA) que, no período pós-seleção, apresentaram aumento do número de ejaculados de baixa sensibilidade (tipo EIII).

Na Tabela 3 está o coeficiente de variação (CV) observado para MOT, MI e NAR, de cada macho, no terceiro e sétimo dias de armazenamento. De um modo geral houve um aumento gradativo no CV com o aumento do tempo de armazenamento. Os menores $\mathrm{CV}$ foram observados para NAR, estando todos abaixo de $20 \%$ no $7^{\circ}$ dia (Tabela 3 ). Os índices de MI apresentaram variação um pouco maior à do NAR, mas inferior à observada para MOT. Os três machos do grupo MAIOR foram os que apresentaram alto CV para MOT, já nas 72h de avaliação, evi- denciando uma maior variação entre seus ejaculados. Machos do grupo MENOR foram os que apresentaram menores CV para MOT (Tabela 3).

Nas Tabelas 4, 5 e 6 estão os percentuais médios de MOT, MI e NAR, respectivamente, para cada macho selecionado. Apesar de terem sido efetuadas avaliações diárias para MOT, somente são apresentados os dados dos dias em que foram avaliados os três parâmetros, de modo a estabelecer um paralelo entre eles. Foi observado, de modo geral, que os machos dos grupos MÉDIA e MENOR não diferem estatisticamente ( $P>0,05)$, em termos de MOT, MI e NAR. Dos machos selecionados para o grupo MÉDIA alguns apresentaram comportamento semelhante aos do grupo MENOR, sendo evidenciado pelos índices de MOT próximos ou acima de $60 \%$, no $7^{\circ}$ dia (machos 6 e 7; Tabela 4).

Não houve correlação significativa $(\mathrm{P}>0,05)$ entre MOT, NAR e MI, na avaliação efetuada nas 24h. Correlações significativas $(\mathrm{P}<0,01)$ entre MOT e MI foram verificadas nas $120(\mathrm{r}=0,59)$ e $168 \mathrm{~h}(\mathrm{r}=0,74)$. Entre MOT e NAR, correlações significativas $(\mathrm{P}<0,01)$ foram observadas nas $72(\mathrm{r}=0,45), 120(\mathrm{r}=0,41)$ e $168 \mathrm{~h}(\mathrm{r}=0,37)$. A correlação entre NAR e MI foi significativa $(\mathrm{P}<0,01)$ nas $72(r=0,37), 120(r=0,43)$ e $168 \mathrm{~h}(r=0,57)$.

Tabela 1. Distribuição dos 30 machos avaliados na pré-seleção de acordo com o número de ejaculados de cada tipo.

\begin{tabular}{ccccccc}
\hline Tipo de & \multicolumn{5}{c}{ Número de machos conforme o número de ejaculados de cada tipo } \\
\cline { 2 - 7 } ejaculado & $\mathbf{0 / 5}$ & $\mathbf{1 / 5}$ & $\mathbf{2 / 5}$ & $\mathbf{3 / 5}$ & $\mathbf{4 / 5}$ & $\mathbf{5 / 5}$ \\
\hline EI & 17 & 6 & 2 & 0 & 2 & 3 \\
EII & 5 & 6 & 3 & 5 & 6 & 5 \\
EIII & 13 & 8 & 4 & 0 & 3 & 2 \\
\hline
\end{tabular}

EI $=$ motilidade $<60 \%$ nas $72 \mathrm{~h}$; EII $=$ motilidade $\geq 60 \%$ nas $72 \mathrm{~h}$ e $<60 \%$ nas $144 \mathrm{~h}$; EIII $=$ motilidade $\geq 60 \%$ nas $144 \mathrm{~h}$

Tabela 2. Número de ejaculados de cada tipo observados nos 12 machos suínos selecionados de acordo com a manutenção da motilidade durante resfriamento do sêmen a $17^{\circ} \mathrm{C}$.

\begin{tabular}{cccccccc}
\multicolumn{2}{c}{ a manutenção da motilidade durante resfriamento do sêmen a $17^{\circ} \mathrm{C}}$. \\
\cline { 2 - 7 } Grupos & \multirow{2}{*}{ Machos } & EI & EII & EIII & EI & Pós-seleção \\
\hline MENOR & 1 & 0 & 1 & 4 & 0 & 1 & EII \\
MENOR & 2 & 0 & 1 & 4 & 0 & 0 & 5 \\
MENOR & 3 & 0 & 1 & 4 & 0 & 2 & 3 \\
MENOR & 4 & 0 & 0 & 5 & 0 & 0 & 5 \\
MENOR & 5 & 0 & 0 & 5 & 0 & 0 & 4 \\
MÉDIA & 6 & 0 & 4 & 1 & 0 & 0 & 4 \\
MÉDIA & 7 & 1 & 4 & 0 & 0 & 1 & 4 \\
MÉDIA & 8 & 0 & 5 & 0 & 1 & 1 & 3 \\
MÉDIA & 9 & 0 & 5 & 0 & 0 & 4 & 0 \\
MAIOR & 10 & 4 & 1 & 0 & 3 & 2 & 0 \\
MAIOR & 11 & 5 & 0 & 0 & 2 & 2 & 1 \\
MAIOR & 12 & 5 & 0 & 0 & 3 & 2 \\
\hline Motilidade <60\% nas & $72 \mathrm{~h}$ (EI), $\geq 60 \%$ nas 72h e <60\% nas 144h (EII) e $\geq 60 \%$ nas $144 \mathrm{~h}$ (EIII). & \\
Mínimo de 4/5 ejaculados EI (MAIOR), EII (MÉDIA) e EIII (MENOR) na pré-seleção. &
\end{tabular}


Tabela 3. Coeficientes de variação da motilidade (MOT), membranas íntegras (MI) e acrossomas normais (NAR) no terceiro e sétimo dia de armazenamento do sêmen de machos suínos selecionados de acordo com a manutenção da motilidade in vitro.

\begin{tabular}{|c|c|c|c|c|c|c|c|}
\hline \multirow{2}{*}{ Grupos } & \multirow{2}{*}{ Machos } & \multicolumn{2}{|c|}{ MOT } & \multicolumn{2}{|c|}{ MI } & \multicolumn{2}{|c|}{ NAR } \\
\hline & & $3^{\circ}$ dia & $7^{\circ}$ dia & $3^{\circ}$ dia & $7^{\circ}$ dia & $3^{\circ}$ dia & $7^{\circ}$ dia \\
\hline MENOR & 1 & $12 \%$ & $57 \%$ & $15 \%$ & $16 \%$ & $1 \%$ & $6 \%$ \\
\hline MENOR & 2 & $6 \%$ & $13 \%$ & $8 \%$ & $10 \%$ & $1 \%$ & $11 \%$ \\
\hline MENOR & 3 & $10 \%$ & $57 \%$ & $7 \%$ & $16 \%$ & $1 \%$ & $3 \%$ \\
\hline MENOR & 4 & $7 \%$ & $11 \%$ & $5 \%$ & $17 \%$ & $3 \%$ & $7 \%$ \\
\hline MENOR & 5 & $10 \%$ & $16 \%$ & $6 \%$ & $9 \%$ & $2 \%$ & $7 \%$ \\
\hline MÉDIA & 6 & $12 \%$ & $67 \%$ & $5 \%$ & $44 \%$ & $1 \%$ & $7 \%$ \\
\hline MÉDIA & 7 & $17 \%$ & $77 \%$ & $9 \%$ & $9 \%$ & $3 \%$ & $4 \%$ \\
\hline MÉDIA & 8 & $15 \%$ & $61 \%$ & $9 \%$ & $21 \%$ & $2 \%$ & $8 \%$ \\
\hline MÉDIA & 9 & $13 \%$ & $212 \%$ & $4 \%$ & $30 \%$ & $2 \%$ & $8 \%$ \\
\hline MAIOR & 10 & $77 \%$ & $316 \%$ & $3 \%$ & $19 \%$ & $2 \%$ & $11 \%$ \\
\hline MAIOR & 11 & $70 \%$ & $197 \%$ & $19 \%$ & $53 \%$ & $3 \%$ & $19 \%$ \\
\hline MAIOR & 12 & $116 \%$ & $316 \%$ & $16 \%$ & $44 \%$ & $7 \%$ & $13 \%$ \\
\hline
\end{tabular}

MENOR = mínimo $4 / 5$ ejaculados MOT $\geq 60 \%$ nas $144 \mathrm{~h}$ na pré-seleção.

MÉDIA = mínimo $4 / 5$ ejaculados MOT $\geq 60 \%$ nas $72 \mathrm{~h}$ e $<60 \%$ nas $144 \mathrm{~h}$ na pré-seleção.

MAIOR= mínimo $4 / 5$ ejaculados MOT $<60 \%$ nas $72 \mathrm{~h}$ na pré-seleção.

Tabela 4. Porcentuais de motilidade espermática (média \pm desvio-padrão) de machos suínos após terem sido selecionados de acordo com a manutenção da motilidade do sêmen armazenado a $17^{\circ} \mathrm{C}$, em BTS.

\begin{tabular}{cccccc}
\hline Grupos & Machos & $\mathbf{1}^{\mathbf{0}}$ dia & $\mathbf{3}^{\mathbf{0}}$ dia & $\mathbf{5}^{\mathbf{0}}$ dia & $\mathbf{7}^{\mathbf{0}}$ dia \\
\hline MENOR & 1 & $87 \pm 4,5 \mathrm{ab}$ & $79 \pm 8,9 \mathrm{a}$ & $73 \pm 6,7 \mathrm{a}$ & $57 \pm 29,7 \mathrm{a}$ \\
MENOR & 2 & $89 \pm 2,2 \mathrm{a}$ & $77 \pm 4,5 \mathrm{a}$ & $71 \pm 4,2 \mathrm{a}$ & $65 \pm 3,5 \mathrm{a}$ \\
MENOR & 3 & $81 \pm 4,2 \mathrm{abc}$ & $73 \pm 5,7 \mathrm{ab}$ & $57 \pm 27,3 \mathrm{ab}$ & $50 \pm 29,4 \mathrm{ab}$ \\
MENOR & 4 & $86 \pm 4,2 \mathrm{ab}$ & $76 \pm 6,5 \mathrm{a}$ & $73 \pm 5,7 \mathrm{a}$ & $63 \pm 8,4 \mathrm{a}$ \\
MENOR & 5 & $86 \pm 2,5 \mathrm{ab}$ & $80 \pm 6,5 \mathrm{a}$ & $75 \pm 7,1 \mathrm{a}$ & $67 \pm 10,4 \mathrm{a}$ \\
MÉDIA & 6 & $81 \pm 4,8 \mathrm{abc}$ & $75 \pm 6,5 \mathrm{ab}$ & $70 \pm 7,1 \mathrm{a}$ & $66 \pm 6,3 \mathrm{a}$ \\
MÉDIA & 7 & $81 \pm 8,9 \mathrm{abc}$ & $76 \pm 7,4 \mathrm{a}$ & $64 \pm 7,4 \mathrm{ab}$ & $58 \pm 19,2 \mathrm{a}$ \\
MÉDIA & 8 & $72 \pm 13,0 \mathrm{bcd}$ & $68 \pm 14,0 \mathrm{abc}$ & $62 \pm 12,0 \mathrm{ab}$ & $50 \pm 13,2 \mathrm{ab}$ \\
MÉDIA & 9 & $85 \pm 5,0 \mathrm{ab}$ & $75 \pm 10,4 \mathrm{ab}$ & $39 \pm 29,5 \mathrm{abc}$ & $0,0 \pm 0,0 \mathrm{c}$ \\
MAIOR & 10 & $63 \pm 20,5 \mathrm{~d}$ & $42 \pm 34,7 \mathrm{bc}$ & $8,0 \pm 17,9 \mathrm{c}$ & $2,0 \pm 4,5 \mathrm{c}$ \\
MAIOR & 11 & $72 \pm 7,6 \mathrm{bcd}$ & $54 \pm 22,5 \mathrm{abc}$ & $34 \pm 31,5 \mathrm{bc}$ & $18 \pm 22,5 \mathrm{bc}$ \\
MAIOR & 12 & $66 \pm 10,8 \mathrm{~cd}$ & $37 \pm 28,4 \mathrm{c}$ & $2,0 \pm 4,5 \mathrm{c}$ & $0,0 \pm 0,0 \mathrm{c}$ \\
\hline
\end{tabular}

MENOR = mínimo $4 / 5$ ejaculados MOT $\geq 60 \%$ nas $144 \mathrm{~h}$ na pré-seleção.

MÉDIA = mínimo $4 / 5$ ejaculados MOT $\geq 60 \%$ nas $72 \mathrm{~h}$ e $<60 \%$ nas $144 \mathrm{~h}$ na pré-seleção.

MAIOR= mínimo $4 / 5$ ejaculados MOT $<60 \%$ nas $72 \mathrm{~h}$ na pré-seleção.

$\mathrm{a}, \mathrm{b}, \mathrm{c}, \mathrm{d}$ na coluna $(\mathrm{P}<0,05)$.

\section{DISCUSSÃO}

Dos 150 ejaculados armazenados e examinados durante a pré-seleção, $22 \%$ apresentaram motilidade inferior a $60 \%$ nas $72 \mathrm{~h}$ enquanto $73 \%$ dos ejaculados estavam com motilidade inferior a $60 \%$ nas $144 \mathrm{~h}$ de armazenamento, confirmando a ocorrência de queda de motilidade ao longo do armazenamento [22, 24].

Considerando os dados das Tabelas 2 e 3, alguns machos apresentaram comportamento diferente do observado no período pré-seleção, em termos de uniformidade dos ejaculados, especialmente nos grupos 
Tabela 5. Porcentuais de membranas espermáticas íntegras (média \pm desvio-padrão) de machos suínos após terem sido selecionados de acordo com a manutenção da motilidade espermática a $17^{\circ} \mathrm{C}$, em BTS.

\begin{tabular}{cccccc}
\hline Grupos & Machos & $\mathbf{1}^{\mathbf{0}}$ dia & $\mathbf{3}^{\mathbf{0}}$ dia & $\mathbf{5}^{\mathbf{0}}$ dia & $\mathbf{7}^{\mathbf{0}}$ dia \\
\hline MENOR & 1 & $78 \pm 1,2$ & $80 \pm 11,7$ & $77 \pm 6,4 \mathrm{ab}$ & $67 \pm 10,6 \mathrm{a}$ \\
MENOR & 2 & $76 \pm 8,6$ & $78 \pm 6,3$ & $77 \pm 7,6 \mathrm{ab}$ & $74 \pm 7,7 \mathrm{a}$ \\
MENOR & 3 & $75 \pm 1,7$ & $72 \pm 4,8$ & $72 \pm 6,0 \mathrm{ab}$ & $64 \pm 10,3 \mathrm{a}$ \\
MENOR & 4 & $77 \pm 8,6$ & $77 \pm 3,5$ & $78 \pm 6,7 \mathrm{ab}$ & $75 \pm 12,9 \mathrm{a}$ \\
MENOR & 5 & $80 \pm 5,4$ & $78 \pm 4,8$ & $77 \pm 13,0 \mathrm{ab}$ & $72 \pm 6,5 \mathrm{a}$ \\
MÉDIA & 6 & $82 \pm 5,0$ & $79 \pm 3,9$ & $81 \pm 3,7 \mathrm{a}$ & $64 \pm 28,5 \mathrm{a}$ \\
MÉDIA & 7 & $71 \pm 8,7$ & $78 \pm 6,7$ & $74 \pm 8,5 \mathrm{ab}$ & $75 \pm 6,5 \mathrm{a}$ \\
MÉDIA & 8 & $74 \pm 9,7$ & $76 \pm 7,1$ & $73 \pm 11,6 \mathrm{ab}$ & $60 \pm 12,8 \mathrm{ab}$ \\
MÉDIA & 9 & $75 \pm 8,4$ & $78 \pm 3,4$ & $55 \pm 10,5 \mathrm{bc}$ & $24 \pm 7,2 \mathrm{c}$ \\
MAIOR & 10 & $77 \pm 7,4$ & $79 \pm 2,5$ & $70 \pm 9,9 \mathrm{ab}$ & $49 \pm 9,7 \mathrm{abc}$ \\
MAIOR & 11 & $76 \pm 5,9$ & $66 \pm 12,6$ & $47 \pm 21,9 \mathrm{c}$ & $50 \pm 26,6 \mathrm{abc}$ \\
MAIOR & 12 & $77 \pm 6,4$ & $67 \pm 10,4$ & $46 \pm 5,1 \mathrm{c}$ & $29 \pm 13,0 \mathrm{bc}$ \\
\hline
\end{tabular}

MENOR = mínimo $4 / 5$ ejaculados MOT $\geq 60 \%$ nas 144 h na pré-seleção.

MÉDIA $=$ mínimo $4 / 5$ ejaculados MOT $\geq 60 \%$ nas $72 \mathrm{~h}$ e $<60 \%$ nas $144 \mathrm{~h}$ na pré-seleção.

MAIOR= mínimo $4 / 5$ ejaculados MOT $<60 \%$ nas $72 \mathrm{~h}$ na pré-seleção.

$\mathrm{a}, \mathrm{b}, \mathrm{c}, \mathrm{d}$ na coluna $(\mathrm{P}<0,05)$.

Tabela 6. Porcentuais de acrossomas normais (média \pm desvio-padrão) de machos suínos após terem sido selecionados de acordo com a manutenção da motilidade do sêmen armazenado a $17^{\circ} \mathrm{C}$, em BTS.

\begin{tabular}{cccccc}
\hline Grupos & Machos & $\mathbf{1}^{\mathbf{0}}$ dia & $\mathbf{3}^{\mathbf{o}}$ dia & $\mathbf{5}^{\mathbf{0}}$ dia & $\mathbf{7}^{\mathbf{0}}$ dia \\
\hline MENOR & 1 & $97 \pm 1,4$ & $96 \pm 0,6 \mathrm{a}$ & $92 \pm 3,3$ & $92 \pm 5,2 \mathrm{ab}$ \\
MENOR & 2 & $97 \pm 1,5$ & $96 \pm 1,1 \mathrm{a}$ & $94 \pm 2,2$ & $88 \pm 9,7 \mathrm{abc}$ \\
MENOR & 3 & $96 \pm 2,0$ & $94 \pm 2,9 \mathrm{ab}$ & $92 \pm 2,8$ & $93 \pm 3,0 \mathrm{ab}$ \\
MENOR & 4 & $97 \pm 1,2$ & $97 \pm 1,7 \mathrm{a}$ & $94 \pm 3,6$ & $93 \pm 6,4 \mathrm{ab}$ \\
MENOR & 5 & $98 \pm 1,0$ & $96 \pm 1,6 \mathrm{a}$ & $94 \pm 3,1$ & $92 \pm 6,0 \mathrm{abc}$ \\
MÉDIA & 6 & $97 \pm 1,4$ & $95 \pm 1,1 \mathrm{ab}$ & $93 \pm 3,1$ & $90 \pm 6,6 \mathrm{abc}$ \\
MÉDIA & 7 & $97 \pm 1,9$ & $95 \pm 2,6 \mathrm{ab}$ & $94 \pm 4,3$ & $94 \pm 4,1 \mathrm{a}$ \\
MÉDIA & 8 & $96 \pm 1,1$ & $96 \pm 2,1 \mathrm{a}$ & $90 \pm 7,8$ & $89 \pm 7,1 \mathrm{abc}$ \\
MÉDIA & 9 & $95 \pm 3,7$ & $94 \pm 1,7 \mathrm{ab}$ & $91 \pm 2,7$ & $82 \pm 6,9 \mathrm{abc}$ \\
MAIOR & 10 & $98 \pm 1,6$ & $95 \pm 1,6 \mathrm{ab}$ & $93 \pm 3,3$ & $85 \pm 9,1 \mathrm{abc}$ \\
MAIOR & 11 & $96 \pm 2,2$ & $94 \pm 2,6 \mathrm{ab}$ & $88 \pm 11,5$ & $79 \pm 14,7 \mathrm{c}$ \\
MAIOR & 12 & $97 \pm 1,6$ & $90 \pm 6,0 \mathrm{~b}$ & $84 \pm 7,8$ & $80 \pm 10,3 \mathrm{bc}$ \\
\hline
\end{tabular}

MENOR = mínimo $4 / 5$ ejaculados MOT $\geq 60 \%$ nas $144 \mathrm{~h}$ na pré-seleção.

MÉDIA = mínimo $4 / 5$ ejaculados MOT $\geq 60 \%$ nas $72 \mathrm{~h}$ e $<60 \%$ nas $144 \mathrm{~h}$ na pré-seleção.

MAIOR= mínimo $4 / 5$ ejaculados MOT $<60 \%$ nas $72 \mathrm{~h}$ na pré-seleção.

$\mathrm{a}, \mathrm{b}, \mathrm{c}, \mathrm{d}$ na coluna $(\mathrm{P}<0,05)$.

MEDIA e MAIOR. Maior variação entre machos do que entre ejaculados do mesmo macho já foi relatada [2], embora com sêmen avaliado apenas durante $5 \mathrm{~h}$ de manutenção a $38^{\circ} \mathrm{C}$, não podendo ser comparada diretamente às avaliações efetuadas durante um longo tempo de armazenamento, como foi o caso do presente estudo. De fato, como comentado anteriormente, o CV aumentou com o tempo de armazenamento, dificultando o processo de seleção, pela diminuição da uniformidade dos ejaculados de um mesmo macho. As razões pelas quais os diferentes ejaculados de um mesmo macho apresentam variação na manutenção da motilidade, 
durante o resfriamento, ainda não são conhecidas. Considerando que espermatozóides ejaculados adquirem maior sensibilidade ao choque térmico ao percorrerem os túbulos epididimários, a diferença na freqüência de coletas e duração do trânsito espermático no epidídimo poderiam contribuir para a variação dos ejaculados de um mesmo macho [23]. A variação entre ejaculados de um mesmo macho também foi observada na capacidade dos espermatozóides em se ligar à zona pelúcida, levando a apresentação da hipótese de que o conteúdo de espermadesinas esteja envolvido [8], o que denotaria, diferenças na composição do plasma seminal de um ejaculado para outro. $\mathrm{O}$ fato das espermadesinas estarem ou não envolvidas com a sensibilidade ao resfriamento, ainda não foi investigado.

Especula-se que a redução na viabilidade espermática in vitro esteja relacionada ao decréscimo dos ácidos graxos polinsaturados, durante o armazenamento do sêmen suíno [4]. A peroxidação dos lipídios da membrana espermática também parece estar envolvida nas alterações estruturais e bioquímicas ocorridas durante o resfriamento [13], levando à perda da permeabilidade seletiva da membrana [6]. No entanto, não há estudos que tenham investigado se estes aspectos diferem entre machos ou entre ejaculados de um mesmo macho.

A diferença de resposta entre ejaculados de um mesmo macho revela a dificuldade em efetuar uma classificação dos machos conforme a sensibilidade ao resfriamento, objetivo do presente estudo. Já foi salientado o fato da avaliação de um único ejaculado não ser um bom indicativo do potencial produtivo de um macho, pois os parâmetros de avaliação seminal apresentam baixa repetibilidade, sendo de 0,21 para a motilidade [14]. Para ter uma estimativa mais precisa do potencial de produção de doses inseminantes, tem sido sugerida a coleta de 9 a 15 ejaculados [14]. No presente estudo, se forem considerados os 10 ejaculados coletados dos 12 machos, nos períodos pré e pós-seleção, aqueles considerados menos sensíveis (grupo MENOR) foram os que apresentaram, no total, um maior número de ejaculados do tipo esperado (Tabela 2).

Embora os valores de MI sejam, de modo geral, superiores aos de MOT, diferenças maiores entre estes dois parâmetros, aparecem no $5^{\circ}$ e $7^{\circ}$ dias para os machos MÉDIA e MENOR. Nos machos MAIOR a queda da MOT é mais acentuada que a da MI, fazendo com que as diferenças entre os dois parâmetros se acen- tuem ao longo do armazenamento (Tabelas 4 e 5). O percentual médio de MI, para todos os machos, nas $24 \mathrm{~h}$ de armazenamento, foi de $77 \%$, similar ao valor de $74 \%$ observado anteriormente [16], logo após a diluição do sêmen.

Os machos apresentaram diferentes percentuais de MOT, em todos os dias de avaliação, mas diferiram em termos de MI, no $5^{\circ}$ e $7^{\circ}$ dias (Tabelas 4 e 5), e de NAR, no $3^{\circ}$ e $7^{\circ}$ dias (Tabela 6 ) de armazenamento. De modo geral, os valores de NAR foram elevados, mesmo após 7 dias de armazenamento. Não foi observado nenhum valor individual de NAR abaixo de $80 \%$, nas $72 \mathrm{~h}$ de armazenamento, mesmo para os machos de MÉDIA ou MAIOR sensibilidade ao resfriamento. Assim, alguns machos que diferiram em termos de MOT apresentaram percentuais de NAR semelhantes (Tabelas 4 e 6).

Cabe salientar que os machos não foram selecionados por diferenças estatísticas em seus parâmetros, mas considerando um valor mínimo de $60 \%$ de motilidade, abaixo do qual pode haver uma queda na eficiência reprodutiva [7]. Se for considerado o mesmo patamar para MI, do total de 228 avaliações efetuadas, compreendendo o $1^{\circ}, 3^{\circ}, 5^{\circ}$ e $7^{\circ}$ dias, 33 valores $(14,5 \%)$ estiveram abaixo de $60 \%$, ao longo do armazenamento. Vinte destes valores foram observados nas $168 \mathrm{~h}$, sendo 11 oriundos de machos de MAIOR sensibilidade e 4 de um macho MEDIA (macho 9). Este macho também apresentou valores de MOT baixos no $5^{\circ}$ e $7^{\circ}$ dias (Tabela 4). Em termos de NAR, $19(8,3 \%)$ valores foram abaixo de $85 \%$. Quinze destes valores foram observados nas $168 \mathrm{~h}$ de avaliação, 7 dos quais oriundos dos machos de MAIOR sensibilidade. Desta forma, os machos selecionados como mais sensíveis ao resfriamento, em termos de MOT, foram também os responsáveis por boa parte dos valores mais baixos de MI e NAR.

A correlação entre parâmetros de avaliação seminal parece controversa pois, em estudo no qual o sêmen suíno foi analisado durante cinco dias de armazenamento a $17^{\circ} \mathrm{C}$, não houve correlação significativa entre MI, NAR e MOT [1], enquanto em outro [26] a correlação foi alta. A existência ou não de correlação entre parâmetros de viabilidade espermática não invalida a utilização de um dos testes, pois vários aspectos estão envolvidos na função espermática, os quais podem ser avaliados por um teste, mas não por outro [11]. A integridade da membrana acrossômica 
não reflete necessariamente a integridade da membrana plasmática [10], a qual parece ser mais lábil [9, 16], o que poderia explicar a ocorrência de espermatozóides com o acrossoma morfologicamente normal mas com a integridade da membrana plasmática e a motilidade alteradas. À medida que as doses de sêmen permaneceram mais tempo armazenadas, a MOT, MI e NAR foram comprometidos, embora em diferente intensidade, o que explicaria o aumento dos valores de correlação, observados no presente estudo, para alguns destes parâmetros, acompanhando o envelhecimento in vitro do sêmen.

\section{CONCLUSÕES}

Machos selecionados como tendo menor sensibilidade espermática ao resfriamento apresentaram menor variação nas características dos ejaculados do período pré- para o pós-seleção. As diferenças observadas entre machos em termos de motilidade nem sempre são acompanhadas por diferenças na integridade do acrossoma ou da membrana espermática.

\section{REFERÊNCIAS}

1 Baltes T.J. 1993. Plasma membrane evaluation with fluorescent stains, and computer-measured motility as indicators of in vitro aging of boar spermatozoa. 102p. Hannover, Alemanha. Tese (Doutorado em Reprodução Animal) - Escola Superior de Hannover.

2 Benson R.W., Pickett B.W., Komarek R.J. \& Lucas J.J. 1967. Effect of incubation and shock on motility of boar spermatozoa and their relationship to lipid content. Journal of Animal Science. 26:1078-1081.

3 Bortolozzo F.P. \& Wentz Ivo. 1997. Sucesso de um programa de IA em suínos. Revista Brasileira de Reprodução Animal. 21:15-21.

4 Cerolini S., Maldjian A., Surai P. \& Noble R. 2000. Viability, susceptibility to peroxidation and fatty acid composition of boar semen during liquid storage. Animal of Reproduction Science. 58: 99-111.

5 Colenbrander B. \& Kemp B. 1991. Factors influencing semen quality in pigs. Journal of Reproduction and Fertility. Suppl 40: 105-115.

6 De Leeuw F.E., Colenbrander B. \& Verkleij A.J. 1991. The role membrane damage plays in cold shock and freezing injury. Reproduction in Domestic Animals. Suppl 1: 95-104.

7 Flowers W.L. 1997. Management of boars for efficient semen production. Journal of Reproduction and Fertility. Suppl 52: 67-78.
8 Harrison R.A.P. 1997. Sperm plasma membrane characteristics and boar semen fertility. Journal of Reproduction and Fertility. Suppl 52:195-211.

9 Harrison R.A.P. \& Vickers S.E. 1990. Use of fluorescence probes to assess membrane integrity in mammalian spermatozoa. Journal of Reproduction and Fertility. 88: 343-352.

10 Holt W.V. 2000. Fundamental aspects of sperm cryobiology: the importance of species and individual differences. Theriogenology. 53: 47-58.

11 Jeyendran R.S. \& Zaneveld L.J.D. 1993. Controversies in the development and validation of new sperm assays. Fertility and Sterility. 59: 726-728.

12 Johnson L.A., Maxwell W.M.C., Dobrinsky J.R. \& Welch G.R. 1996. Staining sperm for viability assessment. Reproduction in Domestic Animals. 31: 37-47.

13 Jones R. \& Mann T. 1977. Damage of ram spermatozoa by peroxidation of endogenous phospholipids. Journal of Reproduction and Fertility. 50: 261-268.

14 Kennedy B.W. \& Wilkins J.N. 1984. Boar, breed and environmental factors influencing semen characteristics of boars used in artificial insemination. Canadian Journal of Animal Science. 64: 833-843.

15 Mariano M.S. 1988. Características biológicas e bioquímicas do sêmen suíno com diferentes resistências à conservação no estado líquido e ao congelamento. 72p. Santa Maria, RS. Dissertação (Mestrado em Fisiopatologia da Reprodução) - Curso de Pós-Graduação em Medicina Veterinária, Universidade Federal de Santa Maria.

16 Ortman K. \& Rodriguez-Martinez H. 1994. Membrane damage during dilution, cooling and freezing-thawing of boar spermatozoa packaged in plastic bags. Journal of Veterinary Medicine. 41: 37-47.

17 Paulenz H., Kommisrud E. \& Hofmo P.O. 2000. Effect of long-term storage at different temperatures on the quality of liquid boar semen. Reproduction in Domestic Animals. 35: 83-87.

18 Pursel V.G., Johnson L.A. \& Rampacek G.B. 1972. Acrossome morphology of boar spermatozoa incubated before cold shock. Journal of Animal Science. 34:278-283.

19 Rodriguez-Martinez H., Zhang B.R. \& Larsson B. 1997. Bovine semen quality and the ability to produce embryos in vivo and in vitro. Arquivos da Faculdade de Veterinária UFRGS. 25: 108-126.

20 SAS Institute. 1998. SAS user's guide: Statistical Analysis System. Cary: SAS Institute, Release 6.12.

21 Vazquez J.M., Martinez E.A., Roca J., Matas C. \& Blanco O. 1998. The fertilizing ability assessment of fresh and stored boar semen. Reproduction in Domestic Animals. 33: $267-270$. 
22 Waberski D., Dirksen G. \& Weitze K.F. 1991. Effect of semen quality on fertility results of stored liquid boar semen - a field trial. Reproduction in Domestic Animals. Suppl 1:375-378.

23 Watson P.F. 1995. Recents developments and concepts in the cryopreservation of spermatozoa and the assessment of their post-thawing function. Reproduction Fertility and Development. 7: 871-891.

24 Weitze K.F. 1991. Long-term storage of extended boar semen. 1991. Reproduction in Domestic Animals. Suppl 1:231-253.

25 Woelders H. 1991. Overview of in methods for evaluation of semen quality. Reproduction in Domestic Animals. Suppl 1: 145-164.

26 Zou C.X. \& Yang Z.M. 2000. Evaluation on sperm quality of freshly ejaculated boar semen during in vitro storage under different temperatures. Theriogenology. 53: 14771488 .

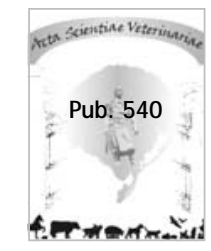

